(C) 2008 IEEE. Reprinted, with permission, from LongBing Cao, Metasynthetic Computing for Solving Open Complex Problems . Computer Software and Applications, 2008. COMPSAC '08. 32nd Annual IEEE International, 2008. This material is posted here with permission of the IEEE. Such permission of the IEEE does not in any way imply IEEE endorsement of any of the University of Technology, Sydney's products or services. Internal or personal use of this material is permitted. However, permission to reprint/republish this material for advertising or promotional purposes or for creating new collective works for resale or redistribution must be obtained from the IEEE by writing to pubspermissions@ieee.org. By choosing to view this document, you agree to all provisions of the copyright laws protecting it 


\title{
Metasynthetic Computing for Solving Open Complex Problems
}

\author{
Longbing Cao
}

\begin{abstract}
The studies of complex systems have been recognized as one of the greatest challenges for the current and future science and technology. Open complex giant systems are a family of specially complex systems with complexities such as openness, human involvement, societal chacteristic and intelligence emergence. They greatly challenge multiple disciplines such as system sciences, cognitive sciences, information sciences, intelligence sciences, and computer sciences. As a result, traditional problem-solving methodologies cannot help with them. The theory of from qualitative to quantitative metasynthesis has been proposed as an breakthrough and effective methodology for the understanding and problem-solving of open complex giant systems. In this paper, we interpret the theory of metasynthesis from the social cognitive interaction perspective. We discuss theoretical framework, problem-solving process, social cognitive evolution, intelligence emergence and pitfalls of certain types of cognitions in developing metasythesisbased problem-solving systems from the perspective of cognitive science and social cognitive interaction. These can enhance the understanding of complex systems, and the design of effective problem-solving systems.
\end{abstract}

\section{INTRODUCTION}

Recently, complex systems have been recognized as one of greatest challenges in the current and future science and technology [28]. As a result, a new scientific field, namely the science of complexity [22], [24], emerges which focuses on the studies of complex systems. This is also evidenced by many emergent research centers of complex systems. As a very special part of the complex system family, open complex giant systems (OCGS) [20], [17] was proposed as a new field. A typical instance of open complex giant systems is Internet [9]. Internet demonstrates system complexities [22] such as open through interactions [27] with environment, giant consisting of billions of hyperlinks, transactions, surfers from every corner of the world, dynamic with fast evolution beyond our imagination, adaptive [12] toward problem-solving and consensus building, uncertain of current state and of the next step, and societal [11] involving human, communities and organizations [21] with varying cultures, tradition, religions, politics, laws, policies and social norms.

The problem-solving of open complex giant systems is very challenging due to their intrinsic system complexities. In fact, many of them are unrecognized or unperceived, for instance, brain informatic working mechanisms. The OCAS problem-solving is highly related to the progress of brain informatics. Furthermore, from the perspective of problemsolving philosophy, we need to consider the cooperation

\footnotetext{
Our thanks to Australian Research Council discovery and linkage grants (DP0667060, DP0773412, LP0775041).

L. Cao is with Faculty of Information Technology, University of Technology, Sydney, Australia lbcaodit.uts.edu.au
}

between human and system, and study what roles human (including brain informatics) can play better in handling OCGS.

In general, the history of human social activities, literature aggradation and explorations in less complex problems have presented us some effective methodologies, philosophies and technologies, which guide us toward understanding an unrecognized and unperceived problem step by step. An empiricial methodological conclusion from such efforts is the establishment of a new field of science: open complex giant systems and its methodology from qualitative to quantitative metasynthesis proposed by some distinguished Chinese Scientists in 1990 [20], [17].

In this paper, from the perspective of social cognitive interaction, we briefly discuss the principles of metasynthesisbased problem-solving for dealing with open complex giant systems. We summarize the framework and process of social cognitive interaction-based metasynthesis, and intelligence emergence for the problem solving. The discussed principles are critical for dealing with system complexities of open complex giant systems, which are one of the greatest challenges in current intelligence sciences, system sciences and cognitive sciences [18].

The remainder is organized as follows. In Section II, we briefly summarize the system complexities of open complex giant systems, and their corresponding challenges to building a problem-solving system. In Section III, a theoretical framework of social cognitive interaction-based metasynthesis is presented. Section IV discusses the metasynthetic problemsolving process on the basis of social cognitive interaction. In Section $\mathrm{V}$, individual cognitive model, social cognitive interaction model, social cognitive intelligent emergence are discussed. We conclude this paper in Section VI.

\section{COMPLEXITIES AND CHALLENGES OF OPEN COMPLEX GIANT SYSTEMS}

System complexities of open complex giant systems consist of openness, giant, hierarchy, human-involvement, societal characteristic, dynamic characteristic, uncertainty, and imprecision, etc. We briefly introduce them in the following paragraphs.

- Openness: An OCGS exchanges energy, information and materials with its external enviornment;

- Giant: An OCGS is composed of hundreds or even millions of system constituents and components;

- Hierarchy: There be usually many levels in an OCGS, in some cases, it is not known how many levels there are in an OCGS; An OCGS consists of many sub-OCGSs, which may further include sub-sub-OCGSs; 
- Human-involvement: Relevant human consist of an essential constituent of an OCGS;

- Societal characteristic: Many social factors such as laws, politics, organizational factors, business processes, are embedded in an OCGS;

- Dynamic characteristic: OCGS is dynamic in the sense that it may change its states, working mechanism, constituents, internal and external interaction mechanism at any time beyond our imagination;

- Uncertainty: At any time point, the system state of OCGS may not be quite clear; in many cases, our understanding of such a system is uncertain that means we do not have a solid and recognizable conclusion about the underlying problem;

- Imprecision: Our understanding of the system is imprecise at a certain stage; such imprecise understanding may continue for quite a long time before a precise one can be obtained.

The above system complexities bring about dramatic challenges to the existing theoretical foundations and technological means in dealing with the problem solving of open complex giant systems. For instance, the following paragraphs list a few such challenges.

- Problem-solving philosophy: Reduction is normally used for decomposing a complex system, while it is not sufficient for handling OCGS; Holism is highlighted in traditional Chinese philosophy; the theory of from qualitative to quantitative metasynthesis advocates the combination of reduction with holism, and build up the so-called systematism [19] as the methodological philosophy;

- Human-machine relationship: Traditionally, we tend to build machine-centered systems such as automated systems; with the increase of system complexities, one realizes the importance of human-machine interaction, while this is not enough for handling OCGS; due to the intrinsic complexities, an OCGS problem-solving system consists of both human (a group of domain experts) and machine components, in which two parties help and collaborate with each other, but human are in control of the problems-solving; we call this "humanmachine-cooperated, but human-centered";

- The power of ubiquitous intelligence: In handling OCGS, ubiquitous intelligence such as human qualitative intelligence, machine quantitative intelligence, social intelligence, domain intelligence, network intelligence are all involved and play different but essential roles in the problem-solving; the problem-solving is a process of multiple types of intelligence interaction and emergence;

- Collective intelligence and social cognitive interaction: In the problem-solving of OCGS, a collection of experienced domain experts and their effective interaction are essentially important; this involves the working mechanisms for social cognitive interaction, group expertbased problem-solving, and the development and emer- gence mechanisms of social intelligence systems etc.;

- Problem-solving methodologies: How to build a problem-solving system for OCGS problems? The answer is to build a Hall for Workshop of Metasynthetic Engineering (HWME); Then, what is an HWME?

- Dynamic system theories: As a problem, OCGS is dynamic; as a problem-solving system, an HWME is dynamic as well; we need to study new dynamic system theories for such systems, and describe the dynamics of system goals, organizational relationships, interaction modes and system states, etc.

From the technical engineering perspective, we also face many challenges, for example:

- Large scale of system simulation and modeling: It is essential to develop simulation tools, lanaguages and evaluation systems to simulate the working mechanism of large scale of social intelligence emergence, group expert interaction-based problem solving;

- Large scale of system analysis and design methods: There is a need of large scale of system analysis and design methodologies, tools and evaluation systems;

- Human-centered computing: How to support domain experts to take the leading problem-solving role in an HWME? how to support dynamic human-machine task allocation and cooperation?

- Metasynthetic computing: It is necessary to combine many types of computing tools

- Knowledge science, engineering and managment: How to capture, represent, transform, discover and use domain knowledge, ad hoc knowledge, meta-knowledge and knowledge from data?

- Online HWME infrastructure: Distributed HWME is necessary because of a wide involvement of problemsolving experts, resources and tools, then how to build such an online HWME?

In the following sections, we try to report our lessons and understanding of the development of problem-solving system from the perspective of social cognitive interaction.

\section{THEORETICAL FRAMEWORK OF SOCIAL COGNITIVE INTERACTION-BASED METASYNTHESIS}

To deal with the system complexities of open complex giant systems, Qian et al. proposed the problem-solving methodology of from qualitative to quantitative metasynthesis [20], [17], [7]. Furthermore, Qian et al. proposed that a feasible and technical solution for the problem-solving of open complex systems is to build a hall for workshop of metasynthetic engineering (HWME). An HWME is a humanmachine-cooperated but human-centered intelligent problemsolving workspace and artificial computing organization. It consists of humans, computers, and relevant computing tools, in which humans help computers and computers support humans. Its key working process is a social cognitive interaction based problem solving, in which all relevant domain experts interact, collaborate, communicate and negotiate with each other like multiple agents toward the problem solving. 
We interpret the theoretical framework of an HWME from system and cognition perspectives. The system framework of social cognitive interaction-based metasynthesis consists of the following key points.

SYSTEM FRAMEWORK: From qualitative to quantitative metasynthesis

- An HWME consists of humans and computers, in which humans and computers are system constituents.

- The capability of an HWME results from the metasynthesis of all system constituents.

- In an HWME, there may emerge many collaborative groups that are formed based on the requirements of problem-solving.

- The members of each group may change with the dynamics of the system and its problem-solving process.

- There is hierarchy in an HWME; some layers are stable, for instance, responsabilitie, roles and permissions; while others may be dynamic.

- An HWME is open in the sense that both iteself and its problem-solving process are dynamic.

- To support the working of HWME, it is necessary to have efficient and detailed index and searching. The resources for indexing are dynamic, some are existing while others may be instantly added by system constituents in the problem-solving process.

- An HWME is capable of receiving messages from its environment.

- There should be effective communications between HWME and its environment, and among the system members of HWME.

- The problem-solving mechanism of an HWME is achieved through the information exchange among system constituents and between an HWME and its environment.

- An HWME needs to provide capabilities such as information storage, accessing, representation, searching, analysis, discovery, inference, transferring, use and management of resources, data, information, metaknowledge and empirical data that may be in qualitative and quantitative, structured and ill-structured forms;

- An HWME must support distributed cooperation and processing, situated perception, effect and inference, run-time internal and external interaction, and dynamic adaptation or control etc.;

In addition, an HWME also involve the following key cognitive characteristics from the perspective of social cognitive interaction.

COGNITIVE FRAMEWORK: Social cognitive interactionbased metasynthesis

- An HWME has goals; goals present charateristics such as hierarchy, relative certainty and dynamic evolution;

- System constituents of an HWME have cognitive capability and social requirements such as beliefs, desires, intentions, reputation, credit, thinking (convergent and divergent), inference, heurism, judgement, self-learning, and learning from others;

- In an HWME, the solving of a problem is through the effective interaction, collaboration and cooperation between human sub-system and a computerized subsystem. In some cases, the problem-solving is humancentred, while for other problems, automated computer systems play major roles;

- Each system constituent has specific cognition, experiences and beliefs about the world; constituents may share their cognition, while there may be none consensus as well;

- A constituent has desires to learn from others, while they can also independently think;

- There are certain rules, norms and policies that must be respected by all system members in the hierarchical job allocation and cooperation;

- There may be domain-specific organizational rules and relationships that must be followed in the problemsolving process of an HWME;

- There is cognitive evolution, restriction, and integration during the cognitive interaction, which help with the consensus building or conflict resolution;

- An HWME is capable of effectively and orderly importing, stimulating, emerging and integrating intelligence, as well as aggregating, summarizing and exporting goals and outputs.

\section{Social COGNITIVE INTERACTION BASED PROBLEM-SOLVING PROCESS}

The fundamental process of metasynthesis based problem solving is shown in Figure 1. Many relevant experts are invited to login in the HWME server of the hall for workshop. They choose respective topics and sessions of their interest or as requested to join in the interaction. The interaction needs to follow certain interaction templates, scripts and protocols. There are two sorts of actions to be taken in the interaction. One is to exchange ideas with other experts online through brainstorm, negotiation or even debate. The other is to call relevant models, methods and computing to simulate and test his/her ideas and hypotheses. As a result, a member generates his/her initial individual results or decisions based on the above discussion and computing results. These are further merged with other members' conclusions through social cognitive interaction and consensus building to form the final problem-solving results. The above basic process of metasynthesis-based problem solving is explained as follows.

BASIC PROCESS: Metasynthesis-based problem solving

1) Define or understand what the problem is, and what are the objectives;

2) Call relevant domain experts to attend the online workshop through the HWME system;

3) Obtain a preliminary understanding of the problem through the online workshop and by involving experts' 


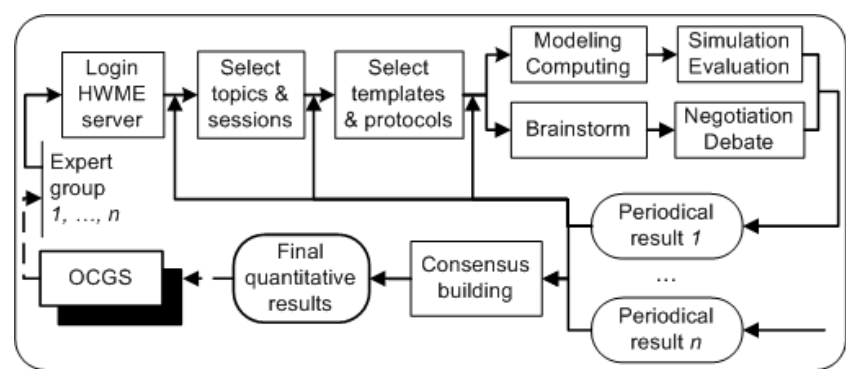

Fig. 1. Principle of hall for workshop of metasynthetic engineering as a problem-solving system.

empiricial knowledge and intuition;

4) Propose solutions for analyzing complex problem structures based on all attendees' expertise and imaginary thinking and the involvement of problem-solving knowledge;

5) Based on the structure characteristic, quantify the problem analysis by involving domain and prior empiricial knowledge progressively and step-by-step;

6) Build quantitative and semi-quantified local or global models for the problem processing; the models come from the involved experts' intelligence and experience, and testify the rules existing in the problem and relevant data;

7) Aggregate or integrate the local/global models into system models if the expert group agrees with the local/global ones;

8) Simulate the system models, and evaluate the models' reliability by involved expert group; if the expert group is not satisfied with the models, go back to step (iii) or any places if necessary to generate more suitable models; the modeling process stops when the involved expert group agrees with the model performance.

Except for the step (i), all other steps are conducted in the online workshop. The key characteristic and function of the online workshop are the social cognitive interaction, collaboration and negotiation among all involved experts, which involve the whole process of problem-solving. Through this process, the individual intelligence is upgraded and aggregated into collective intelligence. In fact, the problem-solving is learning, in particular, group learning based. During the process, all involved experts communicate, argue and negotiate with each other. This not only improves the individual's understanding of the problem, but triggers collective intelligence from the expert group, which outperform the problem-solving capability of individual experts. The collective intelligence plays critical role in solving the problem.

Different from traditional group decision-making, videoconference, network messaging and conference, the social cognitive interaction based metasynthesis is problem-solving oriented, rather than just consensus-building. During the interaction, all relevant data, information, knowledge and data analysis, knowledge discovery and model building are integrated into the HWME system. All these materials and tools consist of relatively complete cyberspace and workplace. In this system, high performance computing of the computer systems, logic reasoning, prior empirical knowledge, and knowledge systems consist of major components of the system. All these resources play important assistant roles in the problem solving.

Complex problem-solving through metasynthesis is a process that all involved experts identify and define problems, specify objectives, design solutions, cognitive integration and intelligence emergence. During this process, it is important to utilize qualitative knowledge, intelligence, domain knowledge, expertise, quantitative computing, network intelligence and computing, social intelligence and computing etc. Another important aspect is interaction environment that supports fair, free and open communication, negotiation, coordination, integration and consensus building of crossdomain and hierarchical group thinking and collective intelligence. We also need proper norms and policies for thinking evolution of effective prevention, deviation rectification, barriar avoidance, stimulus, heurism, infighting and arguement.

From cognitive evolution and intelligence emergence perspective, the following process summarizes the group cognitive evolution for complex problem solving.

COGNITIVE PROCESS: Social coginitive interaction-based cognitive evolution and intelligence emergence.

(1) Open the metasynthesis workshop;

(2) Issue discussion topics;

(3) FOR each topic

(4) Open broad-based discussions of the problem based on brainstorm;

Model initial and target problem status by alternatively through brainstorm and nomination in the expert group; Get the initial approaches and solutions for the problem solving based on brainstorm and Nominal group technique among the expert group;

(6) Get qualitative understanding of the problem through using deep discussion and argument in the expert group with the involvement of the above learned initial approaches, and domain knowledge and experts' intelligence; Form individual HWME discussion sessions focusing on specific topics by dividing the expert group; for each session, try to build semi-quantified and quantitative understanding of the issues on the basis of the above qualitative understanding by using deep discussion and negotiation;

(8) Fuse the outputs from each session into a higher level of preliminary quantitative understanding of the problem through deep discussions and negotiation among the expert group. 
(9) Re-organize the expert group into separate sessions again to structure specific issues identified in the above steps;

(10) Repeat steps (7) and (8) to structure and quantify the problems progressively;

(11) ENDFOR

(12) Aggregate to get the main solutions using methods such as Nominal group technique);

(13) Simulate and evaluate the main solutions by combining computing simulation and deep discussion and negotiation in the expert group;

(14) Review and rank the resulting solutions based on the satisfaction of technical and business expectations, and go back to any step from (2) to (11) if necessary to retrain and refine the solutions;

(15) Obtain the decision-support solutions based on negotiation and nomination in the expert group;

(16) Summarize the qualitative structural principle of the problem solving using methods such as brainstorm and Nominal group technique);

(17) Output the finally agreed findings;

(18) Close the workshop.

\section{Social COGNITIVE INTELLIGENCE EMERGENCE FOR PROBLEM SOLVING}

\section{A. Individual cognitive model}

Individual cognitive capability is defined by three key factors. One is individual cognitive degree, which means to what extent one understands and grasps the whole picture of an object or event. The whole picture may include aspects such as scope, internal dynamics, and external environment. Another factor is object openness degree, which indicates to what extent an object has been completely understood by human being. The third is the individual cognitive methods $v_{i}(i=1, \ldots, N)$, namely how one cognizes an object.

Let $\alpha$ be one's individual cognitive degree, suppose " 1 " reflects $100 \%$ understanding of an object, while " 0 " for nothing. Any value of $\alpha \in[0,1]$ indicates the degree of which one understands the problem. In practice, we often set up a few levels based on qualitative estimation of our understanding of the problem, for instance, "complete", "partial" and "unknown".

Let $\beta$ be the degree of an object openness, suppose "1" indicates a fully solved status, while " 0 " represents unknown. Any value of $\beta \in[0,1]$ reflects the degree of which an problem has been solved. Similarly, we often use words like "well solved", "under-developed", "undeveloped" and "unsolvable" to reflect the openness of a problem. In general, the openness of an object is determined by social cognitive power rather than a particular individual. In some very special cases, for instance, a world-class pioneer coordinates the investigation of a particular issue, his/her individual object openness greatly determines the openness of the problem in the world.

A major individual cognitive method (with $\gamma$ indicating the significance and contribution of method $v_{i}$ to the understanding of underlying problem) is learning. In general, learning can take one of three forms: (1) self-learning, (2) ex-learning, and (3) creative-learning. Self-learning refers to the learning process that is basically ocnducted by the individual's responsability. Ex-learning is driven by the support, supervision, coaching from other senior members. Creativelearning is a process and action that triggers new ideas based on the existing knowledge learned.

The relationship among the three factors is as follows. One understands a problem based on his/her cognitive methods to reach a certain cognitive degree. The individual cognitive degrees of a collection of people determine the level of understanding and problem-solving of the problem. If the collection population approach the most prestigeous group who are specialised in the problem, or if the individual network is extended to the whole contributor society, we then reach the object openness. A person's cognitive state $\tau$ is determined by his/her initial cognitive degree $\alpha$, the degree of an object openness $\beta$, and the contribution $(\gamma)$ of cognitive method used.

$$
\tau=\alpha \beta^{r\left(v_{i}\right)}
$$

In the following sections, we will further discuss social cognitive interaction and cognitive intelligence emergence.

\section{B. Social cognitive interaction model}

For a group of experts, their cognitive methods consist of not only individual learning, but also social interaction. Through the social interaction amongst involved experts, they themselves improve their individual cognitive degree, and at the same time enhance the collective cognitive intelligence as a group about the underlying problem.

Social cognitive interaction consists of key components: social cognitive interaction methods, and group cognitive interaction protocols. Examples of social cognitive interaction methods are "heuristic discussion", "brainstorm" and "debate". They may be organized through a seminar, a workshop, a video conference, or an online workshop or seminar.

Heuristic discussion, or guided discussion, is a form of discussion in which a mentor guides the process and key milestones of each session toward the right directions. The mentor is usually an experienced authority in the field, knows the answer of the problem. His/her role in the discussion is to guarantee that the discussion is productive, efficient and deliverable, and correct any misunderstanding, unnecessary debate or going astray. Brainstorm can encourage the free communications of varying ideas, and is helpful for fostering an enviornment that encourage the emergence of new even conflicting ideas. In a debate, parties may take antagonism position that may be against the reach of any agreement. A more rational debate may build consensus among members and finally reach an agreement. 
There are some key elements concerned in a social cognitive interaction: the number of participants, norms and policies, and interaction protocols. There are a few types of discussions, for instance, peer-to-peer discussion, threeparty discussion, multi-party discussion etc. Members may also be grouped based on their specialism. In some cases, hierarchical groups may be organized to reflect the difference between knowledge and experience, and corresponding role difference in determining the problem solving.

Certain interaction norms, rules and policies are essential for a productive problem solving. We can nominate some rules and policies for an interaction session. They vary as per the background, structure, culture, organizational constraints of involved attendees and the problem openness. For instance, under some situations, the following rules and policies may be recommended to members and groups involved in a problems-solving.

MEMBER NORM: Norms, rules and policies for social cognitive interaction

- Not fully accept anything from an authority; any points need to be checked and evidenced;

- Always think of the conditions of using a concept, conclusion, method, result, etc.;

- Do not use hypotheses without evidence;

- Always believe the limitation and potential of cognition, think of critical and creative ideas.

GROUP NORM: Norms, rules and policies for social cognitive interaction

- Create an equal opportunity for all members, doesn't matter who they are, to nominate new ideas and solutions;

- Any discussions and debate should only be pointed to a specific topic rather than a person or personal characteristics;

- The minority must follow the ideas and solutions coming from the majority;

- Limit the preferences and impacts of senior members at the beginning of a seminar;

- Encourage learning and exchange in an organization and seminar, and respect different viewpoints;

- Criticize a viewpoint if only suitable evidence, conditions and requirements have been found;

As a problem-solving system, certain interaction protocols are needed for social cognitive interaction in an HWME. For example, the following basic protocols may be formed to guide the interaction.

INTERACTION PROTOCOL: Code of conduct for social cognitive interaction
- The lower level of sub-groups must respect the ideas and decisions of a higher one;

- Chairpersons have the authorities to control the process and policies to be used;

- More senior members have higher weights in determining a solution;

- Creative thinking, if recognized by the majority of people, has higher weights in determining a solution.

To support the formulization of social cognitive interaction, we further define a kind of interaction ontology based on descriptive logic. An interaction operator represents a type of interaction mode $\widetilde{m}$ used by relevant members. Examples of interaction operators are as follows.

INTERACTION OPERATOR: Representing interaction mode

- Disjoint $(\widetilde{d})$ : Two sorts of cognitions disjoint from each other;

- Overlap ( $\widetilde{o}$ ): Two sorts of cognitions share some overlap with each other;

- Include $\widetilde{i}$ ): One cognition is a class or part of another.

Interactions following disjoint mode likely lead to disagreement, while modes of overlap and include are more likely converge ideas into a consolidated form.

\section{Cognitive intelligence emergence}

The above defined mechanisms provide a foundation for us to describe cognitive intelligence emergence. Suppose $\tau_{i}$ represent the initial cognitive state of member $i$ on the target problem, member $i$ has authority weight $\mu_{i}$, s/he interacts with other $n$ members by an interaction mode $\widetilde{m}$. In the discussion session, they follow interaction protocols $\wp$. Furthermore, for the interaction trend, let $\wedge$ seek the common points while reserve differences, $\vee$ indicates conflict debate, then we can define a kind of algebra given by the following $\mathrm{BNF}$ to describe the social cognitive interaction:

$$
\mathrm{T}::=0|\mathrm{~T}| \mathrm{T}_{1} \wedge \mathrm{T}_{2} \mid \mathrm{T}_{1} \vee \mathrm{T}_{2}
$$

0 stands for an unknown status about the problem, $\mathrm{T}$ is the cognitive degree determined by one member only, $\mathrm{T}_{1} \wedge \mathrm{T}_{2}$ indicates two members interact toward seeking common points (through convergent thinking), $\mathrm{T}_{1} \vee \mathrm{T}_{2}$ reflects the two members hardly reach an agreement but go opposite ways because of conflict of understanding (divergent thinking).

Thereby we build up the following model describe the social cognitive interaction process and corresponding intelligence emergence from the initial cognitive states of individual members to a resulting state of the problem through social cognitive interaction. The problem-solving degree $\Phi$ is described as follows.

$$
\Phi=\Psi_{j, i=1}^{n}\left(\mathrm{~T} 0, \widetilde{m_{i}}, \wp_{i}, \mu_{i} \tau_{i} \beta^{r\left(v_{i}\right)}\right)
$$


The above model indicates that the current problem-solving status $\Phi$ is an emergent effect of collective cognition accumulated from the interaction among $n$ members based on particular member's initial cognitive state $\tau$, individual $\operatorname{cognitive}$ method $\beta$, the problem openness $\beta$, the weight $\gamma$ of an individual learning method $v$, and the group interaction mode $\tilde{m}$, interaction protocols $\wp$, as well as social impact factors such as a member's cognitive authority weight $\mu_{i}$ and initial cognitive degree of the underlying problem To. Therefore, we say social cognitive problem-solving capabilty is determined not only by initial individual problem-solving degrees, individual interaction modes and authority but also by social cognitive interaction and creative capability.

In addition, the emergence of cognitive intelligence follows a certain cognitive working mechanism formulated by $\Psi_{j} . \Psi_{j}$ reflects the impact of cognitive interaction trend $\mathrm{T}$, interaction protocol $\wp$ and mode $\widetilde{m}$. For instance, for norminal group technique may be used for building consensus of $n$ members.

\section{CONCLUSIONS}

Open complex systems consist of one of the greatest challenges in many areas in current and future research and development, for instance, system sciences, cognitive sciences, intelligence sciences, computer sciences, and information sciences. They are definitely related to brain informatics. One possibly efffective solution is to understand and handle the systems according to the theory of from qualitative to quantitative metasynthesis. Metasynthesis discloses system complexities, human cognitive process, the role difference between humans and machines, and possible directions to go for handling open complex giant systems. In addition, metasynthetic engineering is one of the most suitable techniques for building a problem-solving system in dealing with OCGS. Furthermore, a problem-solving system is a hall for workshop of metasynthetic engineering.

In this paper, we try to understand and interpret the theory of metasynthesis from the social cognitive interaction perspective. We present a picture of social cogntive interactionbased problem solving of open complex systems. Bearing the problem-solving of OCGS in mind, we have tried to link and develop knowledge from multi-disciplines, while highlight the roles and principles of social cognitive interaction-based problem-solving systems. We have introduced the theoretical framework, problem-solving process and intelligence emergence in such systems. With these results, we get a clear picture of the framework, working mechanisms, cognitive interaction models, cognitive evolution and intelligence emergence, and pitfalls of certain types of social cognitive thinking in dealing with open complex problems.

Our future work includes broad and in-depth simulations of social cognitive interaction among large scale of domain experts. We will consider varying background, the study of comprehensive collective thinking methods and positioning, and working mechanism of interaction protocols and intelligence emergence.

\section{REFERENCES}

11] L.B. Cao, Study on issues in agent-based open giant intelligent systems, PhD thesis, Chinese Academy of Sciences, 2002.

[2] Cao, L.B., \& Dai, R.W., Open complex intelligent systems, Post and Telecom Press, 2008.

[3] Cao, L.B., Zhang, C.Q., \& Dai, R.W., Organization-Oriented Analysis of Open Complex Agent Systems, Int. J. on Intelligent Control and Systems, 2005, 10(2):114-122.

[4] Cao, L.B., Zhang, C.Q., \& Dai, R.W., The OSOAD Methodology for Open Complex Agent Systems, Int. J. on Intelligent Control and Systems, 10(4):277-285, 2005.

[5] Cao, L.B., Zhang, C.Q., \& Zhou, M.C., Engineering open complex agent systems: a case study, IEEE Trans. On Systems, Man, and Cybernetics, Part C, 2008.

[6] Cui, X., Study on WWW-based model design in hall for workshop of metasynthetic engineering, $\mathrm{PhD}$ thesis, Chinese Academy of Sciences, 2004.

[7] Dai, R.W., From qualitative to quantitative metasynthetic engineering, Pattern recognition and artificial intelligence, 6(2):60-65, 1993.

[8] Dai, R.W., Wang, J., \& Tian, J., Metasynthesis of intelligent systems, Zhejiang Science and Technology Press, 1995.

[9] Dai, R.W., \& Cao, L.B., Internation: an open complex giant system, Science in China (Series E), 33(4), 289-296, 2003.

[10] Gao, H.X., Study on knowledge reconstruction methods in hall for workshop of metasynthetic engineering, $\mathrm{PhD}$ thesis, Chinese Academy of Sciences, 2003.

[11] Giddens, A., Duneier, M., \& Appelbaum, R.M., Essentials of Sociology, New York. W.W. Norton \& Company, 2006.

[12] Holland, J.H., Hidden Order: How Adaptation Builds Complexity, Washington,D.C, USA:Perseus Books/Publishing, 1995.

[13] Janis, I.L., Victims of Groupthink, Boston. Houghton Mifflin Company, 1972.

[14] Janis, I.L., Groupthink, Psychological Studies of Policy Decisions, Mifflin, Boston, MA., 1982.

[15] Li, Y.D., Study on the design and implementation of hall for workshop of metasynthetic engineering, PhD thesis, Chinese Academy of Sciences, 2003.

[16] Li, X., \& Dai, R.W., Conceptual system structure and metasynthesis, Technical report, Chinese Academy of Sciences, 1998.

[17] Qian, X.S., Re-study on open complex giant systems, Pattern recognition and artificial intelligence, 4(1):5-8, 1991.

[18] Qian, X.S., On Cognitive Sciences, Shanghai Science and Technology Press, 1996.

[19] Qian, X.S., Building systematism, Xishang Science and Technology Press, 2001.

[20] Qian, X.S., Yu, J.Y., \& Dai, R.W., A new scientific field-open complex giant systems and the methodology, Chinese Journal of Nature,13(1),3-10,1990.

[21] Robbins, S.P., Organization Behavior:concepts,controversies and applications(7th ed.), Prentice Hall Inc.,1996.

[22] Waldrop, M., Complexity, USA:Simon and Schuster,1992.

[23] Wang, D.L., Group thinking in hall for workshop of metasynthetic engineering, Technical Report, Chinese Academy of Sciences, 2001.

[24] Warfield, J.N., Twenty Laws of Complexity:Science Applicable in Organization.Systems Research and Behavioral Science ,1998,16(6):1-38.

[25] Warfield, J.N., Warfield, J.N. (2006) An Introduction to Systems Sciences, World Scientific, Singapore.

[26] Warfield, J.N., \& Teigen, C., Groupthink, Clanthink, Spreadthink and Linkthink, Institute for Advanced Study of the Integrative Sciences, George Mason University, Fairfax, VA., 1993.

[27] Wegner, P., Why Interaction is More Powerful than Algorithms, Communications of the ACM,40(5):80-91, 1997.

[28] Weaver, W., Science and Complexity, American Scientist, 36: 536, 1948. 